\section{Forscher kreieren Abspeckmittel}

Es senkt den Blutzuckerspiegel, bremst den Appetit und fördert die Kalorienverbrennung. Das Dreifachhormon, das Forscher entwickelt haben, kann auf diese Weise das Körpergewicht um ein Viertel senken, vor einer Leberverfettung bewahren und die Cholesterinwerte im Lot halten - bisher allerdings nur bei Mäusen. Das Team hat ein Peptid gebastelt, das gleich an drei verschiedenen Punkten im metabolischen Netzwerk angreift, und zwar an den Rezeptoren für GLP-1, GIP und Glucagon. GLP-1 und GIP gehören zum Inkretinsystem, sie werden in Gegenwart von Glukose im Darm freigesetzt und erhöhen die Sekretion von Insulin aus den Betazellen.

Finan B et al. Nature Medicine2014, doi:10.1038/nm.3761

\section{Joghurt senkt Diabetes-Risiko}

Wissenschaftler haben untersucht, was es mit dem Diabetesschutz durch Joghurt auf sich hat: Es zeigte sich eine inverse Beziehung. Je mehr Joghurt die untersuchten Personen konsumierten hatten, desto seltener erkrankten sie an Typ-2-Diabetes. In einer Metaanalyse von 14 prospektiven Kohorten mit 459.790 Teilnehmern sank das Risiko um 18\% je zusätzlicher Portion Joghurt pro Tag. Für das, was als Portion definiert war, benötigt man indes einen größeren Löffel. Denn sofern in den Studien Mengenangaben verfügbar waren, betrug die Portion Joghurt 244 g. In Deutschland enthält ein Becher Joghurt üblicherweise eine Menge von $150 \mathrm{~g}$.

Chen M et al. BMC Medicine 2014: 12: 215

\section{Keine starren Zielvorgaben!}

Metformin? Sulfonylharnstoffe? Glitazone? Oder doch lieber gleich Insulin? Die Medikamentenvielfalt in der Diabetestherapie scheint schier unendlich und sogar Leitlinien betonen, dass unterschiedliche Vorgehensweisen möglich sind. Experten empfehlen die gemeinsame Vereinbarung individueller Therapieziele zwischen Arzt und Patient. Konkrete Beispiele finden Sie neben vielen weiteren spannenden Themen in unserem Schwerpunkt „Diabetes".

\section{Claudia Daniels} Redakteurin

\section{Frühe Intensivtherapie lohnt sich auch nach 20 Jahren noch}

Die Studiengruppe des Diabetes Control and Complications Trial zu den Effekten der intensivierten Insulintherapie von Typ-1-Diabetikern hat Follow-up-Daten vorgelegt, mit Fokus auf Komplikationen an den Augen. Sie zeigen: Auch 23 Jahre nach Studienende zahlt sich die sechseinhalbjährige Phase der Intensivtherapie noch aus. Für die Analyse nahmen sich die Wissenschaftler die Häufigkeit von Augenoperationen vor, die aufgrund von Diabeteskomplikationen erforderlich wurden.
Solche Eingriffe benötigten 63 (8,9\%) von 711 Patienten, die sich 23 Jahre zuvor einer intensivierten Therapie unterzogen hatten. In der Gruppe der 730 konventionell Therapierten mussten sich $98(13,4 \%)$ an den Augen operieren lassen. Insgesamt ergab sich rechnerisch eine Senkung des Risikos für diabetesbedingte Eingriffe an den Augen um $48 \%$ zugunsten der intensiv behandelten Patienten.

DCCT/EDIC Research Group. N Engl J Med 2015; 372: 1722

\title{
Gestationsdiabetes: Langzeitrisiken für Mutter und Kind
}

Der Gestationsdiabetes ist nicht nur ein Schwangerschaftsrisiko, sondern sowohl für Mutter als auch Kind mit Langzeitrisiken assoziiert. Eine Nachbeobachtung von Frauen mit einem Gestationsdiabetes zeigte, dass nach im Durchschnitt sechs Jahren post partum $12 \%$ an einem Typ-2-Diabetes erkrankt waren und fast $20 \%$ entweder eine gestörte Nüchternglykämie oder eine Glukosetoleranzstörung aufwiesen. Darüber hinaus ist auch das Risiko für eine postpartale Depression signifikant erhöht. Aber nicht nur bei der Mutter, sondern auch beim Kind muss mit Langzeitfolgen gerechnet werden. So kann es zu einer Fehlprogrammierung der neuroendokrinen Systeme und des Pankreas kommen, was wiederum zu Adipositas, einem metabolischen Syndrom und schließlich einem Typ-2-Diabetes führen kann.

Symposium "Gestationsdiabetes und Typ-2-Diabetes bei Frauen", 50. Deutscher Diabeteskongress, Berlin 2015

\section{Schutz vor Depressionen}

Depressionen sind für Diabetespatienten gefährlich, weil sie die Stoffwechselerkrankung häufig verschlechtern. Ein Forschungsinstitut hat deshalb das KurzzeitProgramm „DIAMOS - Diabetesmotivation stärken" speziell für Diabetiker entwickelt, die Anzeichen einer erhöhten Depressivität zeigen. Die Wirksamkeit der Kurzzeit-Verhaltenstherapie wurde jetzt in einer Studie belegt, berichtet die Deutsche Diabetes Gesellschaft. An der Studie nahmen 214 Patienten mit Typ-1- oder Typ2-Diabetes teil. Nach zwölf Monaten analysierten die Forscher, wie sich die DIAMOS-Kurzzeittherapie gegenüber einer einer Kontrollgruppe mit Diabetesschulung auf die Teilnehmer ausgewirkt hatte: „Sowohl die Diabetesschulung als auch die DIAMOS-Kurzzeittherapie verbesserten das Selbstmanagement und die Zufriedenheit der Patienten in vergleichbarem Maße“, bilanzierte PD Bernhard Kulzer. „Aber was die Reduktion depressiver Symptome und diabetesbezogener Belastungen betrifft, war DIAMOS eindeutig effektiver als die Schulung." So lag die Wahrscheinlichkeit, eine behandlungsbedürftige Depression zu entwickeln, bei den Teilnehmern der Kurzzeit-Therapie um 37\% niedriger.

www.aerztezeitung.de 